\title{
Demonstrative experiment study on the consistency of two-slit interference and diffraction phenomenon
}

Jie Zhao, Dayong Wang, Yunxin Wang, Lu Rong, Zhuqing Jiang, et al.

Jie Zhao, Dayong Wang, Yunxin Wang, Lu Rong, Zhuqing Jiang, Yuhong Wan, "Demonstrative experiment study on the consistency of two-slit interference and diffraction phenomenon," Proc. SPIE 9793, Education and Training in Optics and Photonics: ETOP 2015, 979318 (8 October 2015); doi: $10.1117 / 12.2223103$

Event: Education and Training in Optics and Photonics: ETOP 2015, 2015, Bordeaux, France 


\title{
Demonstrative experiment study on the consistency of two-slit
}

\section{interference and diffraction phenomenon}

\author{
Jie Zhao ${ }^{\mathrm{a}}$, Dayong Wang ${ }^{*}$, Yunxin Wang ${ }^{\mathrm{b}}$, Lu Rong ${ }^{\mathrm{b}}$, Zhuqing Jiang ${ }^{\mathrm{b}}$, Yuhong Wan ${ }^{\mathrm{b}}$

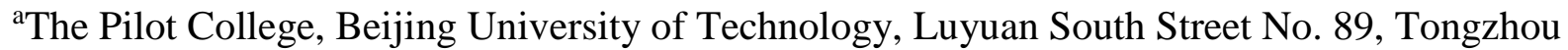 \\ District, Beijing, China 101101; ' Institute of Information Photonics Technology and College of \\ Applied Sciences, Beijing University of Technology, Ping Leyuan No. 100, Chaoyang District, \\ Beijing, China 100124
}

\begin{abstract}
In the Optics of University Physics, two most important concepts are interference and diffraction, which reflect the wave nature of the light. The corresponding content is relatively abundant, such as Young's Double-slit Interference, One-slit Diffraction, Grating Diffraction. But they are not easy for undergraduates to comprehend. So in order to show the two phenomenon visually, the related demonstrative experiment equipment are well-developed, but they are independent with each other. The students can accept them separately, but in the same time ignore the connection between them two. Actually, interference and diffraction are consistent in essence from the view of coherent superposition and redistribution of the light intensity. The difference only depends on the specific parameters. The objective of our paper is to analyze the condition for two slits to produce interference and diffraction phenomenon. Also, apply the knowledge of Fourier Optics to analyze the Fresnel diffraction. This would be a good example for the theory of grand unification in physics. Firstly, the intensity distribution is deduced for two-slit interference, one-slit Fraunhofer diffraction, two-slit Fraunhofer and Fresnel diffraction applying complex-amplitude integration method. In the same time, the simulated experimental results by MATLAB are shown. Secondly, the experimental results are given to verify the analysis. Finally, a new idea is presented to realize an equipment of demonstrative experiment for teaching.
\end{abstract}

Keywords: Interference, diffraction, consistency, demonstrative experiment

\section{INTRODUCTION}

In the course of University Physics, there is an important part, which is Optics. The content is mainly about the wave optics, which has played the important role in kinds of fields, such as interference measurement ${ }^{[1-4]}$, digital microscopic holography ${ }^{[5-9]}$, three-dimensional display ${ }^{[10-12]}$ et al. The content for this part of course is arranged as Young's double-slit interference, one-slit Fraunhofer diffraction and grating diffraction, which are related with interference, diffraction and interference\& diffraction. And the fundamental knowledge is interference and diffraction. Different methods are adopted to analyze. In the view of interference, the optical path difference is calculated, and the relationship is built with the constructive interference and destructive interference. However, half-wavelength band method is applied to describe the one-slit Fraunhofer diffraction. So it seems like they are different from each other. However, in essence they are same on analyzing the redistribution of light intensity, which is caused by coherent superposition. The coherent superposition

*wdyong@bjut.edu.cn; phone 86-10-67391741; fax 86-10-67391741

Education and Training in Optics and Photonics: ETOP 2015, edited by Eric Cormier, Laurent Sarger Proc. of SPIE Vol. 9793, 979318 - (C) 2015 SPIE, IEEE, OSA, ICO · doi: 10.1117/12.2223103 
happens between two or limited light for interference, but infinite sub-light for diffraction. To be precise, if the optical beam obeys the geometrical optics to propagate in straight line, then the superposition is purely about interference, such as Film Interference. Otherwise, interference and diffraction exist in the same time, such as Young's two-slit experiments.

The two concepts are hard for the students to comprehend, because of their abstract character, also knowledge reserve is acquired, such as coherence, optical path, phase et al. Therefore, the demonstrative experiments have been well-developed to show the phenomenon, further for application. For example, Michelson Interferometer is brought to measure the micro displacement, refractive index or thickness of medium, Wedge Film and Newton's ring are used to test the flatness of the optical elements, and Film Interference is applied to realize reflection reducing and increasing effect. There are also lots of similar simulation work completed by optical software. However, it could be easier for students to understand after they have the idea of the consistency of interference and diffraction.

On behalf of this, this paper focus on the typical phenomenon, which is the Young's two-slit experiment. And analyze the condition for two-slit interference and two-slit diffraction phenomenon to show that interference and diffraction is the same in essence only depending on specific parameters. Simulation by MATLAB and experimental results verify the conclusions. Some paper discuss about this ${ }^{[13,14]}$, but the experimental parameters are somewhat vague, or mention little about Fresnel diffraction, which would be discussed in this paper.

\section{PRINCIPLE AND SIMULATION RESULTS}

\subsection{Young's two-slit interference}

The schematic of Young's two-slit interference experiment is shown in Fig. 1 . $S$ is one slit, $S_{1}$ and $S_{2}$ are two same slits, which are parallel to $\mathrm{S}$ and same distance away. The distance between $\mathrm{S}_{1}$ and $\mathrm{S}_{2}$ is $d$. $\mathrm{E}$ is the screen to observe the interference fringes. $D$ is the distance between $\mathrm{E}$ and two-slit screen. $P$ is a point on $\mathrm{E}$ with coordinate $x$. The optical path difference between two beams is $\delta=r_{2}-r_{1}$. Because $D$ is about $10^{4}$ times larger than $d$, the angle $\theta$ is very small. In the condition of approximation, $\delta$ can be described as Eq. (1):

$$
\delta=r_{2}-r_{1} \approx d \sin \theta \approx d \tan \theta=\frac{x d}{D} .
$$

And the appropriate phase difference is $\Delta \varphi$, as shown in Eq. (2):

$$
\Delta \varphi=\frac{2 \pi}{\lambda} \delta \approx \frac{2 \pi}{\lambda} \frac{x d}{D} .
$$

$\lambda$ is the wavelength. The intensity distribution on the screen $I$ is as Eq. (3):

$$
I=I_{1}+I_{2}+2 \sqrt{I_{1} I_{2}} \cos \Delta \varphi,
$$

and the intensity of the two beams $I_{1}, I_{2}$ is the same as $I_{0}$. So $I$ can be simplified as Eq. (4):

$$
I \approx I_{0}+I_{0}+2 \sqrt{I_{0} I_{0}} \cos \left(\frac{2 \pi}{\lambda} \frac{x d}{D}\right)=4 I_{0} \cos ^{2}\left(\frac{\pi d}{\lambda D} x\right)
$$




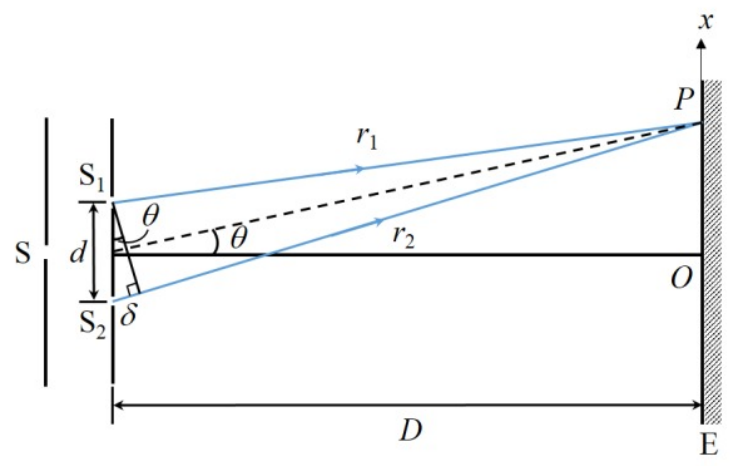

Fig. 1 The schematic of Young's two-slit interference experiment.

Here $I_{0}$ is assumed to be unit as ' 1 '. $D$ is $1 \mathrm{~m}, d$ is $0.2 \mathrm{~mm}$, and is $532 \mathrm{~nm}$. The profile of the intensity on the screen simulated by MATLAB is shown as Fig. 2. It is like parallel, equidistant, dark-and-light.

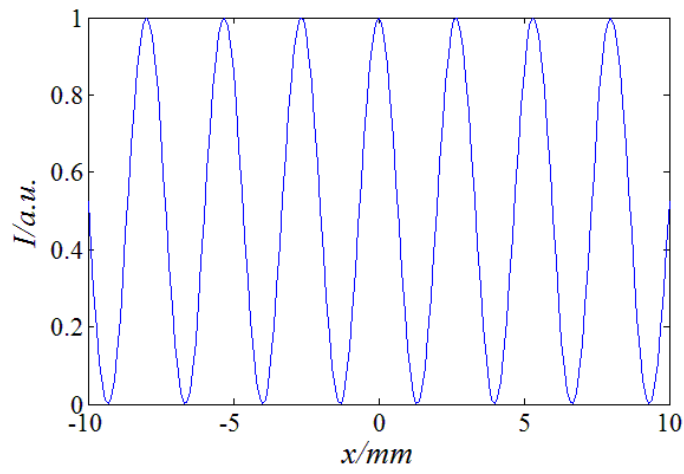

Fig. 2 The simulated profile of the intensity on the screen of Young's two-slit interference experiment.

\subsection{One-slit Fraunhofer diffraction}

Following, it is about the diffraction. When one-slit Fraunhofer diffraction is studied, the schematic is described as Fig. 3. The spherical wave produced by point source $\mathrm{S}$ is collimated by lens $\mathrm{L}_{1}$. Then go through one slit, which is on $\mathrm{K}$ screen, and its size is $a$. After that, the diffracted beams are collimated by lens $\mathrm{L}_{2}$. The Fraunhofer diffraction pattern shows on $\mathrm{E}$ screen. $f$ is the focal length of $\mathrm{L}_{1}$ and $\mathrm{L}_{2} . \quad \theta$ is the diffraction angle.

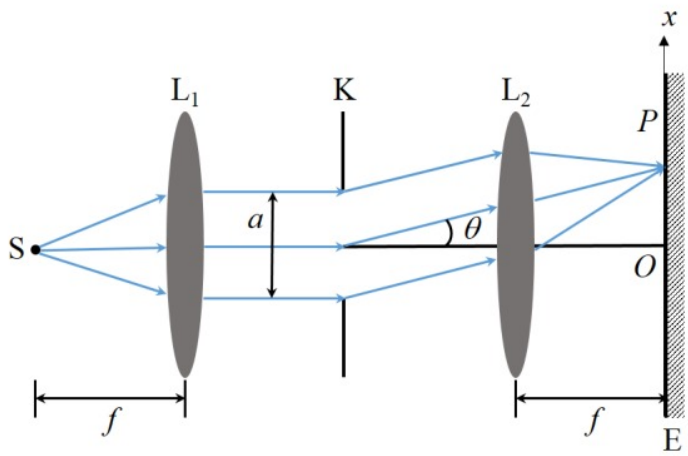

Fig. 3 The schematic of one-slit Fraunhofer diffraction experiment. 
In order to analyze the intensity distribution on E plane, amplitude vector method is used to obtain the light intensity, shown in Fig. 4. According to Huggens-Fresnel principle, it is assumed that the slit is composed with $N$ subwave point sources, which are distributed evenly. Each subwave has the amplitude $r$ and phase difference between them is $\varphi_{0}$. In the triangle $\mathrm{BCD}$, the amplitude of the synthetic vector $A$ can be obtained as Eq. (5):

$$
A=\sqrt{R^{2}+R^{2}-2 R^{2} \cos \left(N \varphi_{0}\right)}=2 R \sin \frac{N \varphi_{0}}{2} .
$$

While in the triangle BCE, the amplitude $r$ can be similarly expressed as Eq. (6):

$$
r=2 R \sin \frac{\varphi_{0}}{2}
$$

So $A$ can be transformed to Eq. (7):

$$
A=\frac{r \sin \frac{N \varphi_{0}}{2}}{\sin \frac{\varphi_{0}}{2}} .
$$

Because $\varphi_{0}$ is very small, so $A$ can be approximated to Eq. (8):

$$
A \approx r N \frac{\sin \frac{N \varphi_{0}}{2}}{\frac{N \varphi_{0}}{2}} .
$$

Then the intensity $I$ should be as Eq. (9):

$$
I=A^{2} \approx I_{0} \frac{\sin ^{2} \alpha}{\alpha^{2}} .
$$

$I_{0}=(N r)^{2}$ is the intensity of the zero order, and $\alpha=\frac{N \varphi_{0}}{2}$.

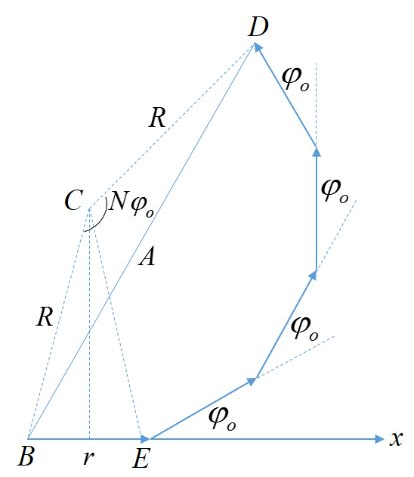

Fig. 4 Schematic of the amplitude vector method to analyze one-slit Fraunhofer diffraction.

For the case of one slit, $N \varphi_{0}$ is the maximum phase difference, which is related with the maximum light path difference $a \sin \theta . \quad \theta$ is small. So $\alpha$ can be written as Eq. (10):

$$
\alpha=\frac{\pi a \sin \theta}{\lambda} \approx \frac{\pi a x}{f \lambda} \text {. }
$$


Here $I_{0}$ is assumed to be unit as ' 1 '. $a$ is $2 \mu \mathrm{m}, f$ is $150 \mathrm{~mm}$, and $\lambda$ is $532 \mathrm{~nm}$. The profile of the intensity on the screen simulated by MATLAB is shown as Fig. 5. It is symmetrical, and the central intensity is much bigger than surroundings.

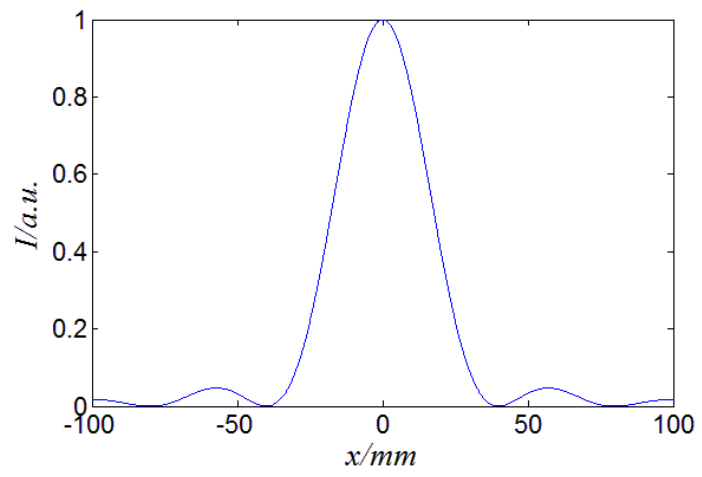

Fig. 5 The simulated profile of the intensity on the screen of one-slit Fraunhofer diffraction experiment.

\subsection{Two-slit Fraunhofer diffraction}

Then, it is about two-slit Fraunhofer diffraction, whose schematic is shown in Fig. 6. $a$ is the size of the slit, $b$ is size of the screen between two slits, and $d=a+b$.

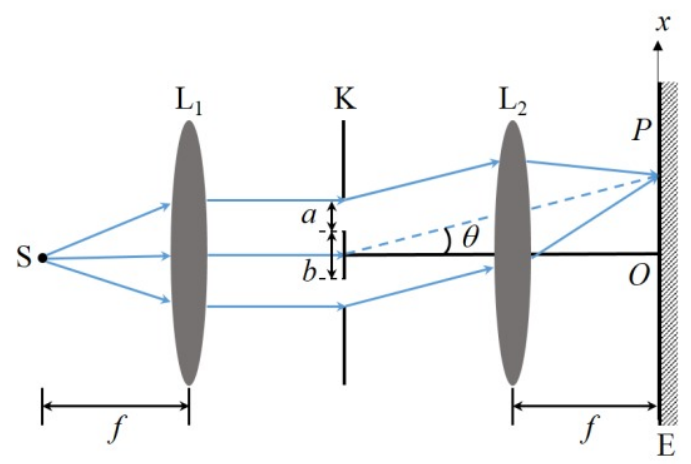

Fig. 6 The schematic of two-slit Fraunhofer diffraction experiment.

The intensity on the screen $\mathrm{E}$ is deduced following two-slit interference, which is expressed as Eq. (11):

$$
I=4 I_{0}^{\prime} \cos ^{2} \frac{\Delta \varphi}{2}
$$

And $I_{0}^{\prime}$ is the intensity of one-slit diffraction, which is written as Eq. (12)

$$
I_{0}^{\prime}=I_{0} \frac{\sin ^{2} \alpha}{\alpha^{2}}
$$

So $I$ can be described as Eq. (13):

$$
I=4 I_{0} \frac{\sin ^{2} \alpha}{\alpha^{2}} \cos ^{2} \frac{\Delta \varphi}{2}
$$

$I_{0}$ is assumed to be unit as '1', $\alpha \approx \frac{\pi a x}{f \lambda}$, and $\Delta \varphi=\frac{2 \pi}{\lambda} d \sin \theta \approx \frac{2 \pi d}{\lambda}-x$ in the case of small diffraction angle. $a$ is $0.6 \mu \mathrm{m}, d$ is $1.8 \mu \mathrm{m}, f$ is $150 \mathrm{~mm}$, and $\lambda$ is $532 \mathrm{~nm}$. The profile of the intensity simulated by MATLAB on the screen is 
shown as Fig. 7. The curve in dotted line is the profile of one-slit diffraction, which modulate the intensity of two-slit interference, and realize the result of two-slit diffraction (solid line). It is the relevance between them three.

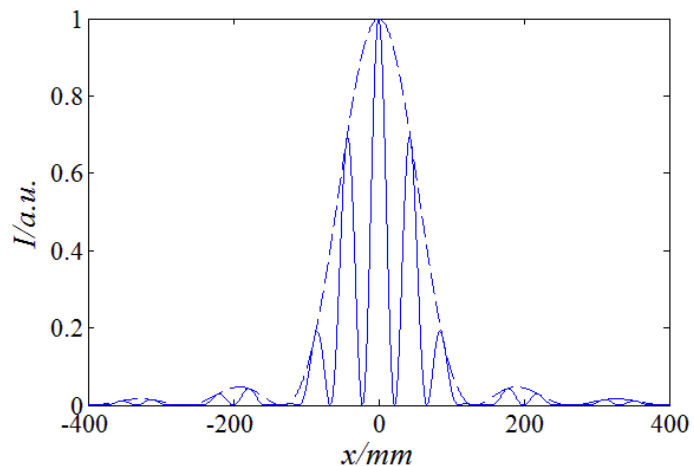

Fig. 7 The simulated profile of the intensity on the screen of two-slit Fraunhofer diffraction experiment, solid line: two-slit Fraunhofer diffraction, dotted line: one-slit Fraunhofer diffraction.

The essence of the difference between interference and diffraction lies on the parameters. If $a$ is changed from $0.6 \mu \mathrm{m}$ to $0.3 \mu \mathrm{m}, 0.1 \mu \mathrm{m}, 0.05 \mu \mathrm{m}$ and $0.01 \mu \mathrm{m}$, while remain others parameters, the profile of two-slit Fraunhofer diffraction seems like alerting to the profile of two-slit interference, as shown in Fig. 8.

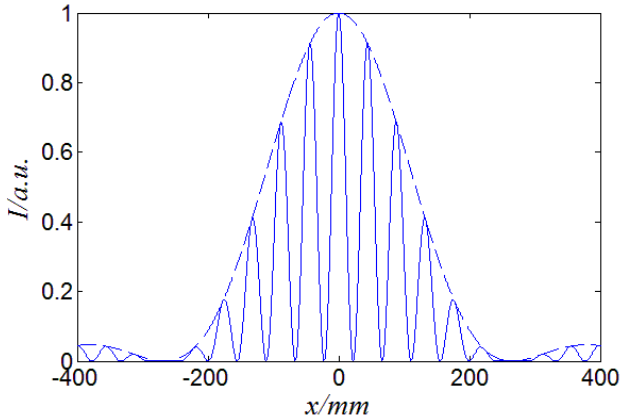

(a)

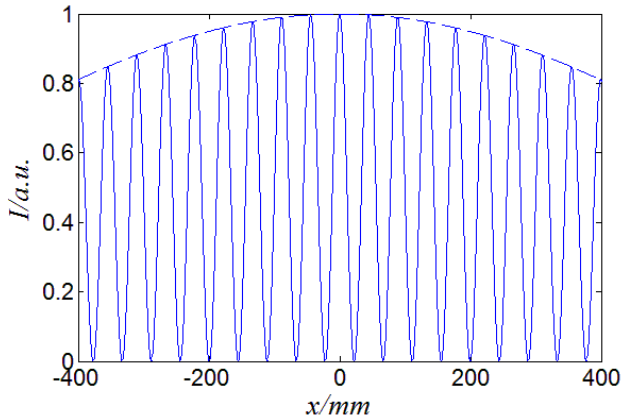

(c)

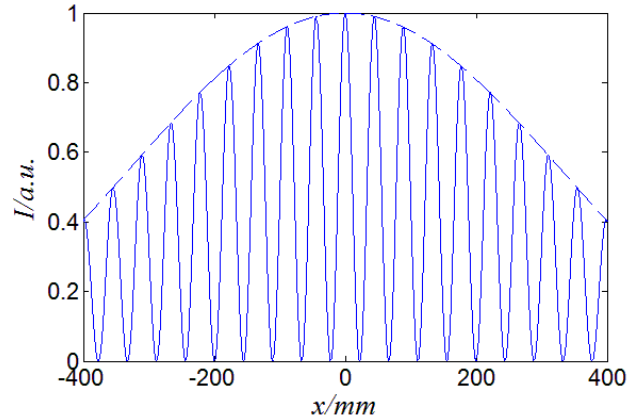

(b)

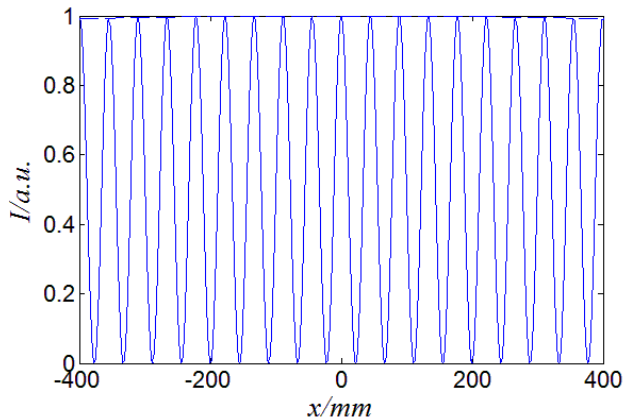

(d)

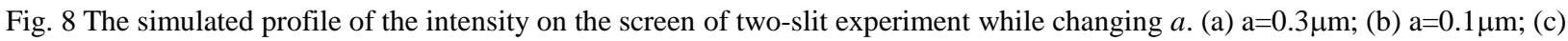
$\mathrm{a}=0.05 \mu \mathrm{m} ;(\mathrm{d}) \mathrm{a}=0.01 \mu \mathrm{m}$. 


\subsection{Two-slit Fresnel diffraction}

It is based on two-slit Fraunhofer diffraction, only the lens behind two-slit screen is taken off, and the schematic is shown as Fig. 9.

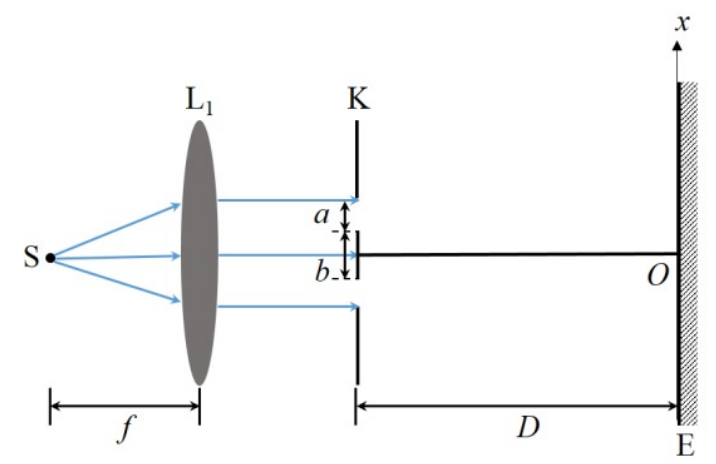

Fig. 9 The schematic of two-slit Fresnel diffraction experiment.

It is assumed that light with unite amplitude incidents on $\mathrm{K}$ vertically. $a$ is $200 \mu \mathrm{m}, d$ is $320 \mu \mathrm{m}, D$ is $115 \mathrm{~mm}$, and is 532nm. The intensity on screen $\mathrm{E}$ is simulated by applying Fresnel diffraction formula, shown in Eq. (14):

$$
\varphi(x, y)=\exp (-j k D) \frac{j k}{2 \pi D} \exp \left[-\frac{j k}{2 D}\left(x^{2}+y^{2}\right)\right] F F T 2\left\{\varphi\left(x_{0}, y_{0}\right) \exp \left[-\frac{j k}{2 D}\left(x_{0}^{2}+y_{0}^{2}\right)\right]\right\}
$$

$\left(x_{0}, y_{0}\right)$ and $(x, y)$ are the coordinate on $\mathrm{K}$ and $\mathrm{E}$ plane separately, $\varphi\left(x_{0}, y_{0}\right)$ is the transmission function of the two-slit, $k$ is the wave vector, and FFT2 refers two-dimensional Fast Fourier transform. When $D$ is $1000 \mathrm{~mm}$, the result of Fresnel diffraction is similar as Fraunhofer diffraction, which is described as Eq. (15):

$$
\varphi(x, y)=\exp (-j k D) \frac{j k}{2 \pi D} \exp \left[-\frac{j k}{2 D}\left(x^{2}+y^{2}\right)\right] F F T 2\left\{\varphi\left(x_{0}, y_{0}\right)\right\}
$$

Fig. 10 is the simulated results of two-slit Fresnel and Fraunhofer diffraction, which show their differences. The pixel size on E plane $(\Delta x, \Delta y)$ can be calculated by Eq. (16):

$$
\Delta x=\frac{\lambda D}{N \Delta x_{0}}, \Delta y=\frac{\lambda D}{N \Delta y_{0}}
$$

$\left(\Delta x_{0}, \Delta y_{0}\right)$ is the pixel size and $N$ is sampling number on $\mathrm{K}$ plane.

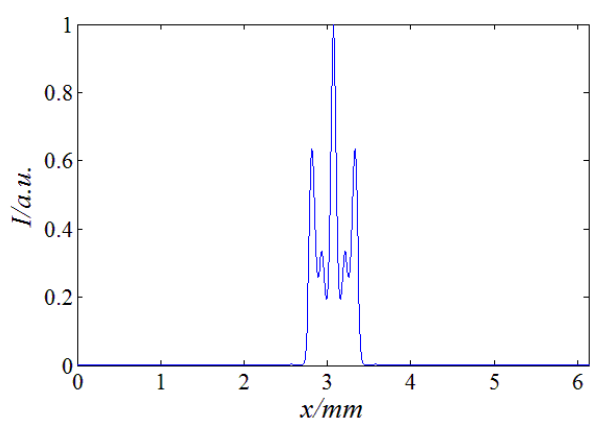

(a)

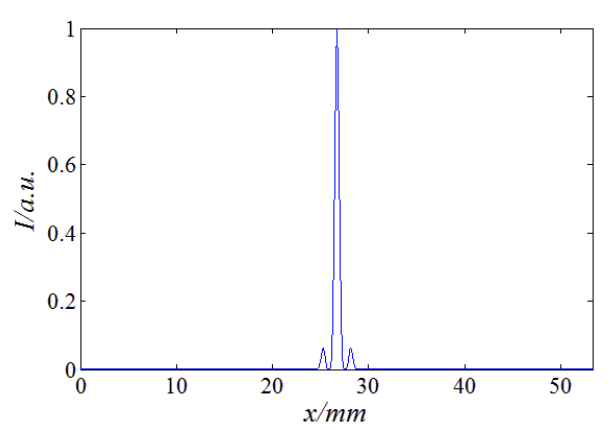

(b)

Fig. 10 The simulated profile of the intensity on the screen of two-slit Fresnel diffraction and Fraunhofer diffraction. (a) Two-slit Fresnel diffraction, (b) two-slit Fraunhofer diffraction. 


\section{EXPERIMENTAL RESULTS}

In this part, the experimental results are shown in Fig. 11, which are recorded by CCD. The used objects include one-slit with size of $100 \mu \mathrm{m}$, two-slit with size of $100 \mu \mathrm{m}$ and distance of $160 \mu \mathrm{m}$, two-slit with size $52 \mu \mathrm{m}$ and distance of $94 \mu \mathrm{m}$. The propagation distance is around $200 \mathrm{~mm}$. They are in agreement on the simulated results, which are the one-slit diffraction, two-slit Fresnel diffraction and two-slit interference separately. The result especially of two-slit interference is far from the expected one, and the reason is that the size of the slit is not small enough and the diffraction efficiency is also low.

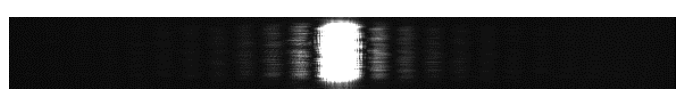

(a)

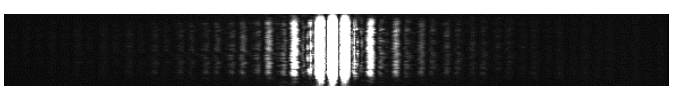

(b)

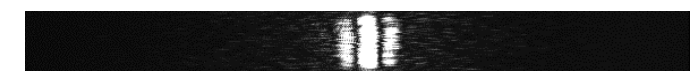

(c)

Fig. 11 Experimental results of the intensity on the screen of one-slit diffractioin, two-slit Fresnel diffraction and two-slit interference.

(a) One-slit diffraction, (b) two-slit Fresnel diffraction, (c) two-slit interference.

So the new idea is to device a two-slit mechanic element, shown in Fig. 12. The distance between two slits and the size of one slit can be adjusted and measured, which are both $0-1 \mathrm{~mm}$ and the step is $1 \mu \mathrm{m}$. It can be used to demonstrate the two-slit interference and diffraction phenomenon when alert the parameters continuously. Till now, no company has been found to do this, which is the future work.

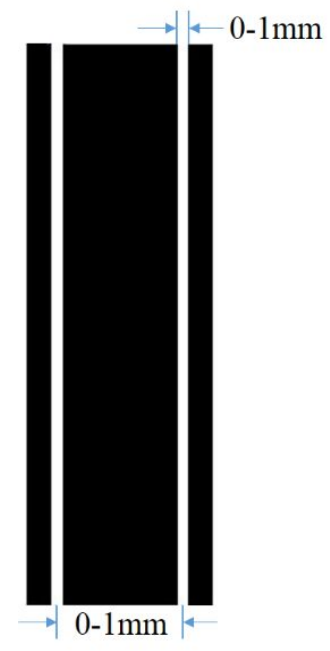

Fig. 12 The schematic of the devised adjusted two-slit mechanic element.

\section{CONCLUSIONS}

In this paper, the principle of two-slit interference, one-slit diffraction and two-slit diffraction is introduced. Also the simulated and experimental results are given. The point is to show the connection between them. When the size of the slit is reduced, two-slit interference turns to two-slit diffraction, whose intensity is modulated by the profile of one-slit 
diffraction. The difference of Fraunhofer and Fresnel diffraction is also analyzed, applying the knowledge of Fourier Optics. Besides, a new idea of devising an mechanic element of two-slit is presented, and can be an important component of a teaching demonstrative equipment to show the consistency of two-slit interference and diffraction. This paper is helpful for the undergraduates to realize the essence of interference and diffraction, and the equipment based on will be a visual tool to assist.

\section{ACKNOWLEDGEMENT}

This work is financially supported by the National Natural Science Foundation of China (No. 61475011, 61307010 and 61205010), the Research Fund for the Doctoral Program of Higher Education of China (No. 20121103120003), Science and Technology Project of Beijing Municipal Commission of Education (No. KM201310005031), the Open Project of Beijing Municipal Engineering Research Center (No. Q5024000201301), the Importation and Development of High-Caliber Talents Project of Beijing Municipal Institutions (CIT\&TCD201504020), Young Talent Project of Beijing University of Technology, the Fundamental Research Fund of Beijing University of Technology (No. X4024000201501), and the Special Matching Project of Local Supported by Centre.

\section{REFERENCES}

[1] Javidi B., Tajahuerce E., "Three-dimensional object recognition by use of digital holography," Opt. Lett. 25(9), 610-612(2000).

[2] Mir M., Wang Z., Shen Z., et al, "Optical measurement of cycle-dependent cell growth," PNAS 108(32), 13124-13129(2011).

[3] Hlubina P., Kadulova M., Ciprian D., et al, “Temperature sensing using the spectral interference of polarization modes in a highly birefringent fiber," Opt. Laser Eng. 70, 51-56(2015).

[4] Armendáriz G., Cravioto-Lagos J., Velázquez V., et al. Teaching quantum mechanics with the Hong-Ou-Mandel interferometer. Proc. of SPIE, 9289, 928908-1-7(2014).

[5] Goodman J. W. and Lawrence R. W., "Digital image formation from electronically detected holograms," Appl. Phys.

Lett. 11, 77-79(1967).

[6] Kim M. K., "Principles and techniques of digital holographic microscopy," SPIE Reviews 1, 018005-1-50(2010).

[7] Kemper B. and Bally G. von, "Digital holographic microscopy for live cell applications and technical inspection," Appl. Opt., 47(4), A52-61(2008).

[8] Colomb T., Cuche E., Charrière F., et al, "Automatic procedure for aberration compensation in digital holographic microscopy and applications to specimen shape compensation,” Appl. Opt., 45(5), 851-863(2006).

[9] Miccio L., Alfieri D., Grilli S., et al, "Direct full compensation of the aberrations in quantitative phase microscopy of thin objects by a single digital hologram,” Appl. Phys. Lett., 90, 041104-1-3(2007).

[10] Teng D. D., Liu L. L., Wang Z. X., et al, "Interactive holographic three-dimensional display with a spatial mouse," Opt. Commun. 306, 121-127(2013).

[11] Hwang Y. S., Bruder F. K., Facke T., et al, "Time-sequential autostereoscopic 3-D display with a novel directional 
backlight system based on volume-holographic optical elements," Opt. Express. 22(8), 9820-9838(2014).

[12] Kakue T., Tahara T., Ito K., et al, "Parallel phase-shifting color digital holography using two phase shifts," Appl. Opt., 48(34), H244-250(2009).

[13] Tang Y. L., Hu G., Zhang J., "From a double-slits experiment to see the essence of optical interference and diffraction (in Chinese)," Physical Experiment of College 24(3), 35-38(2011).

[14] Huang R., Zhang J. X., Liu Z. C., et al, "The comparison of double-slit interference and double-slit diffraction based on the MATLAB (in Chinese)," Physical Experiment of College 28(1), 90-92(2015). 